\title{
Electrochemical selection and characterization of a high current-generating Shewanella oneidensis mutant with altered cell-surface morphology and biofilm-related gene expression
}

\author{
Atsushi Kouzuma ${ }^{1 *}$, Hitomi Oba', Nozomi Tajima ${ }^{2,4}$, Kazuhito Hashimoto ${ }^{2,3}$ and Kazuya Watanabe
}

\begin{abstract}
Background: Shewanella oneidensis MR-1 exhibits extracellular electron transfer (EET) activity that is influenced by various cellular components, including outer-membrane cytochromes, cell-surface polysaccharides (CPS), and regulatory proteins. Here, a random transposon-insertion mutant library of S. oneidensis MR-1 was screened after extended cultivation in electrochemical cells (ECs) with a working electrode poised at $+0.2 \mathrm{~V}(\mathrm{vs}$. Ag/AgCl) to isolate mutants that adapted to electrode-respiring conditions and identify as-yet-unknown EET-related factors.

Results: Several mutants isolated from the enrichment culture exhibited rough morphology and extraordinarily large colonies on agar plates compared to wild-type MR-1. One of the isolated mutants, designated strain EC-2, produced 90\% higher electric current than wild-type MR-1 in ECs and was found to have a transposon inserted in the SO_1860 (uvrY) gene, which encodes a DNA-binding response regulator of the BarA/UvrY two-component regulatory system. However, an in-frame deletion mutant of SO_1860 (_SO_1860) did not exhibit a similar level of current generation as that of EC-2, suggesting that the enhanced current-generating capability of EC-2 was not simply due to the disruption of SO_1860. In both EC-2 and $\triangle$ SO_1860, the transcription of genes related to CPS synthesis was decreased compared to wild-type MR-1, suggesting that CPS negatively affects current generation. In addition, transcriptome analyses revealed that a number of genes, including those involved in biofilm formation, were differentially expressed in EC-2 compared to those in $\Delta \mathrm{SO}_{-} 1860$.
\end{abstract}

Conclusions: The present results indicate that the altered expression of the genes related to CPS biosynthesis and biofilm formation is associated with the distinct morphotype and high current-generating capability of strain EC-2, suggesting an important role of these genes in determining the EET activity of S. oneidensis.

Keywords: Extracellular electron transfer, Microbial fuel cell, Anode respiration, Bioelectrochemical systems

\section{Background}

Shewanella species, which are affiliated with the class Gammaproteobacteria, are widely distributed in nature, including marine, freshwater, sedimentary, and soil environments [1]. Members of this genus are able to respire various organic and inorganic compounds (e.g., oxygen, fumarate, nitrate, nitrite, thiosulfate, and elemental sulfur), as well as soluble and solid metals (e.g., iron, manganese,

\footnotetext{
* Correspondence: akouzuma@toyaku.ac.jp

'School of Life Sciences, Tokyo University of Pharmacy and Life Sciences,

1432-1 Horinouchi, Hachioji 192-0392, Tokyo, Japan

Full list of author information is available at the end of the article
}

uranium, chromium, cobalt, technetium, and vanadium) [2-5]. In recent years, a few Shewanella species have attracted considerable attention due to their potential applicability for bioremediation [6] and bioelectrochemical systems (BESs), such as microbial fuel cells (MFCs) and microbial electrosynthesis cells [7-10].

Shewanella oneidensis MR-1 is the most extensively studied strain in the genus Shewanella because of its metabolic versatility [11], annotated genome sequence $[12,13]$, and ease of genetic manipulation [14]. In addition, since it was shown in 1999 that strain MR-1 has the ability to transfer electrons to an extracellular electrode without 
exogenously added mediator [7], it has served as a model bacterium for studying microbial current generation and extracellular electron transfer (EET) pathways $[1,15]$. These studies have revealed that MR-1 cells have multiple EET pathways, including direct EET pathways that involve outer-membrane cytochromes (OM-cyts) [15] and electrically conductive pilus-like structures (nanowires) [16,17], and indirect EET pathways that function via self-produced electron shuttle compounds, such as flavins [18-21].

Despite these studies, a deeper understanding of the molecular mechanisms of EET is required to optimize and enhance microbial electron transfer rates in BESs because studies in S. oneidensis MR-1 have also indicated that EET is a complex process that is influenced by various intracellular and extracellular components. Saffarini et al. [22] and Charania et al. [23] have revealed that cyclic AMP (cAMP) and CAMP receptor proteins are necessary for the expression of OM-cyts. In addition, Covington et al. [24] identified the $u s h A$ gene, which is involved in flavin secretion in MR-1. Our previous studies have also suggested the possibility that extracellular components, including cell-surface polysaccharides (CPSs), are involved in EET and current generation in BESs $[25,26]$. It is therefore conceivable that many unknown factors are also involved in EET.

A useful approach for identifying unknown cellular components (and genes) associated with a particular phenotype involves the construction and screening of random mutant libraries for mutants with altered phenotypes. We previously succeeded in isolating MR-1 mutants with increased current-generating activities from a transposon (Tn) insertion mutant library that was cultured under electrode-respiring conditions [25,26]. Notably, the obtained mutants had an altered (rough) colony morphology on agar plates [25,26], indicating that the electrochemical cultivation and subsequent screening on agar plates of random Tn insertion mutants is a useful approach for isolating current-generating mutants. Although our previous studies have identified several genes related to cell-surface morphology and current generation [25,26], it is reasonable to speculate that many other unknown factors remain to be identified, considering the complexity of bacterial cell-surface structures and EET processes.

In the present study, we screened MR-1 mutants from a random Tn insertion library for altered colony morphology on agar plates after selection in electrochemical cells (ECs), to identify mutants with enhanced current-generating capability. One of the isolated mutants, designated strain EC2, formed extraordinary large colonies on agar plates and generated higher electric current in an EC than wild-type (WT) MR-1. Analyses of strain EC-2 revealed that a number of genes, including those involved in CPS synthesis and biofilm formation, were differentially expressed compared to WT, suggesting that these genes were associated with the distinct phenotype of this mutant.

\section{Results}

\section{Electrochemcial selection of mutants}

A library of $S$. oneidensis MR-1 random transposon mutants was introduced into an EC reactor equipped with a working electrode poised at $+0.2 \mathrm{~V}(\nu s . \mathrm{Ag} / \mathrm{AgCl})$ and cultivated under electrode-respiring conditions. A current versus time curve generated during the electrochemical cultivation of MR-1 is shown in Additional file 1: Figure S1. After 40-days of electrochemical cultivation, the electrolyte was sampled spread on agar plates for the isolation of mutant cells. We found that approximately $20 \%$ of colonies formed by the electrochemically cultivated mutants were larger than those of WT MR-1, suggesting that the mutants with large-colony morphology grew preferentially under the electrode-respiring conditions.

One of the isolated mutants, designated strain EC-2, was clearly distinct from the other mutants and WT strains as it formed an extraordinary large colony (Additional file 2: Figure S2 and Figure 1). We previously found that a CPS-deficient mutant of MR-1, $\triangle S O \_3177$, formed large, flat, and rough colonies on agar plates, and generated higher power output in MFCs compared to that of WT [25], indicating an association between cell-surface morphology and current generation. Strain EC-2 also exhibited a rough, flat colony morphology, but formed larger colonies than those of $\Delta \mathrm{SO} \_3177$ (Figure 1). Strain EC-2 was therefore selected for further characterization, including genetic analyses, to determine the identity and function of the mutated gene(s) in this mutant.

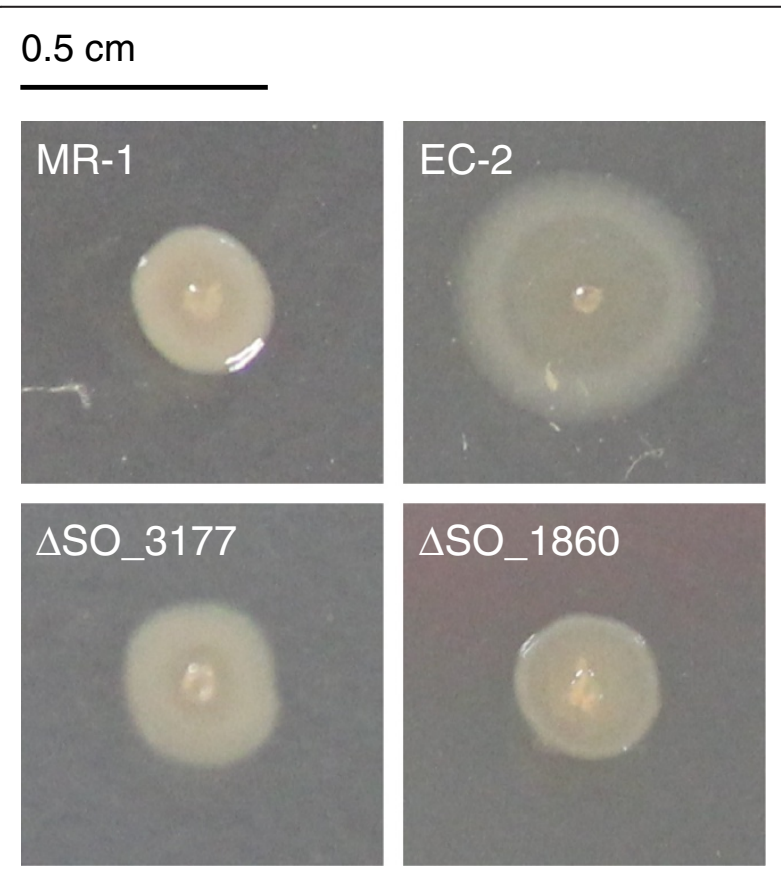

Figure 1 Colony morphology of $S$. oneidensis MR-1 and mutant strains on LB agar plates. 


\section{Evaluation of strain EC-2 in MFC}

The ability of strain EC-2 to generate electrical power in an MFC was analyzed and compared with that of WT (current versus time curves are shown in Additional file 3: Figure S3). To evaluate MFC performance, polarization (Figure 2A) and power (Figure 2B) curves were determined for each MFC after the current generation became stable (day 14 in Additional file 3: Figure S3). The $P_{\max }$ and $I_{\mathrm{sc}}$ for the EC-2 MFC $\left(8.74 \mu \mathrm{Wcm}^{-2}\right.$ and $62.7 \mu \mathrm{Acm}^{-2}$, respectively) were approximately 1.5 -fold higher than those for the WT MFC $\left(6.46 \mu \mathrm{Wcm}^{-2}\right.$ and $41.3 \mu \mathrm{Acm}^{-2}$, respectively).

In the single-chamber MFC used in this study, Shewanella cells were localized to either the electrolyte as planktonic cells, or to the anode and cathode, where they formed biofilms. For strain EC-2, the turbidity of the MFC electrolyte was markedly lower than that in the WT MFC (data

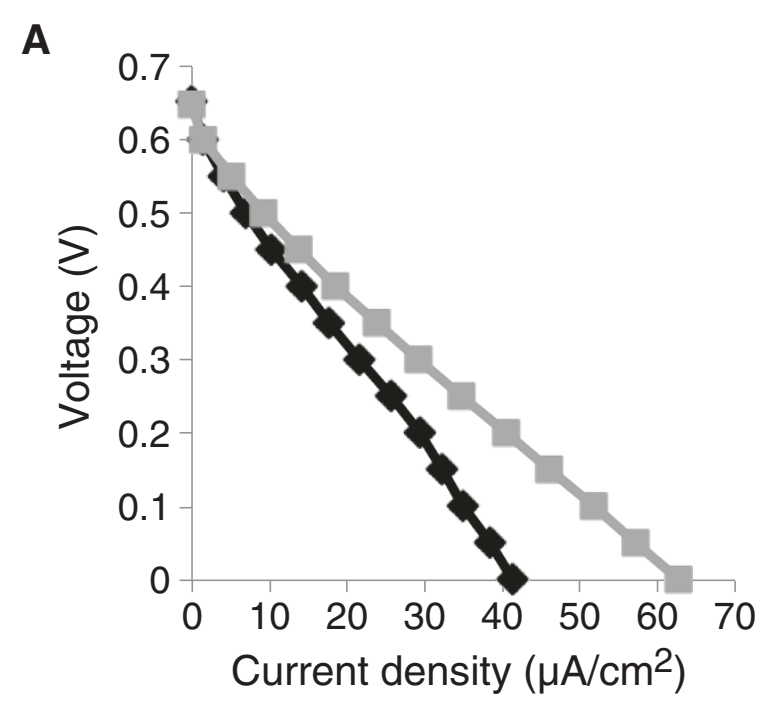

B

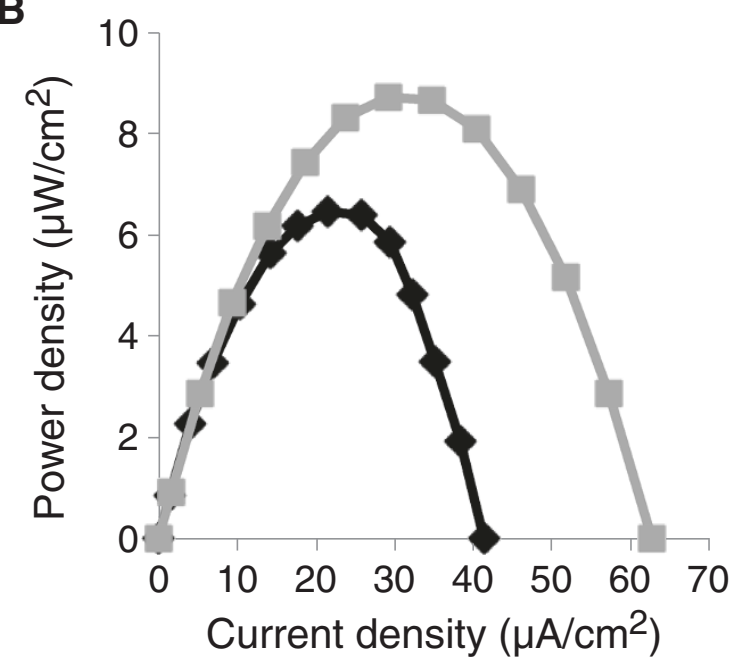

Figure 2 Evaluation of strain EC-2 in MFCs. (A) Polarization and (B) power curves for MFCs containing strain EC-2 (grey line) and wild-type (WT) MR-1 (black line) are shown. not shown), suggesting that more mutant cells were attached to the electrodes. To examine this hypothesis, WT and EC-2 cells from the electrolyte and electrodes of each MFC were collected after approximately $360 \mathrm{~h}$ of operation, and the protein contents in these samples were measured to estimate cell concentration (Figure 3). In the EC-2 MFC, the amount of cells attached to the graphite felt anode $(53.0 \pm 10.2 \mathrm{mg})$ was $140 \%$ higher than that of WT cells $(22.5 \pm 2.4 \mathrm{mg})$, whereas the amount of EC-2 cells in the electrolyte $(7.6 \pm 0.3 \mathrm{mg})$ was $74 \%$ lower than that of WT cells $(29.2 \pm 1.1 \mathrm{mg})$. The total protein contents, which represented the sums of the protein contents in the three MFC areas, did not significantly differ between the two strains. Together, these results indicate that strain EC-2 has the increased ability to adhere to the graphite felt anode when cultivated under electrode-respiring conditions.

\section{Identification of the Tn-insertion site in strain EC-2}

PCR analysis of strain EC-2 revealed that a Tn was inserted into the SO_1860 gene, whose deduced amino acid sequence showed a significant homology (70\% identity) to the DNA-binding response regulator UvrY of Escherichia coli. In E. coli, UvrY is part of the BarA/UvrY two-component regulatory system [27], which regulates the Csr (carbon storage regulation) system via transcriptional activation of CsrB, a small noncoding RNA [28,29]. It has been reported that the BarA/UvrY/Csr regulatory cascade in E. coli is involved in the regulation of numerous physiological functions, including carbon metabolism, motility, adhesion, and biofilm formation $[28,29]$. Recent studies also revealed that the BarA/UvrY/Csr regulatory cascade is conserved in S. oneidensis MR-1, and that SO_1860 (UvrY) is involved in the transcriptional regulation of more than 200 genes,

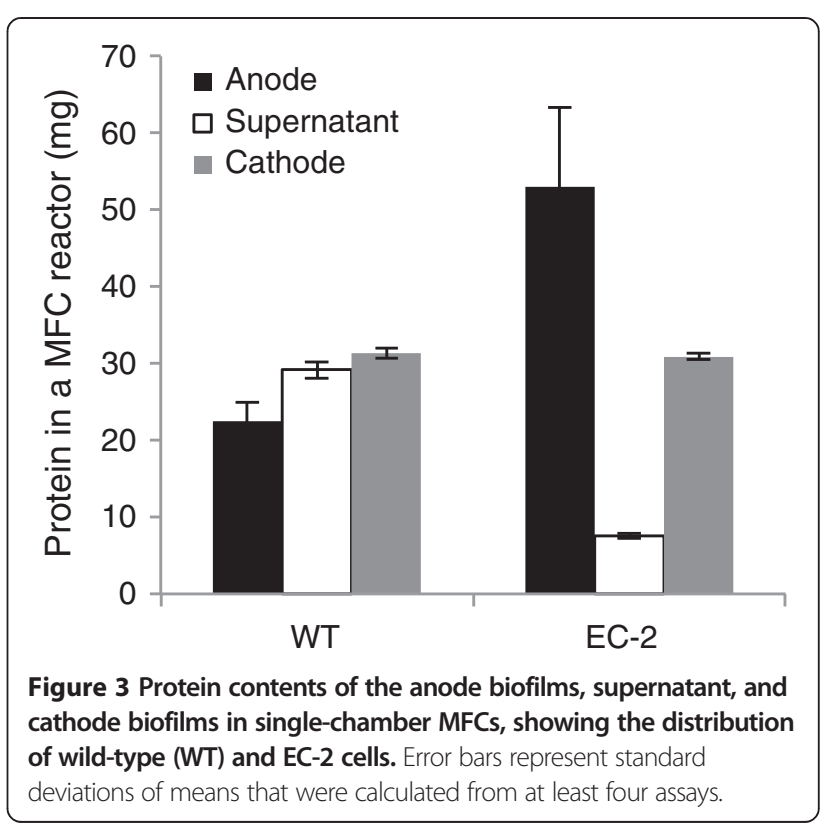


including those involved in CPS biosynthesis genes [30] and biofilm formation [31]. However, the roles of SO_1860 in EET and current generation have not yet been investigated.

\section{Deletion of the SO_1860 gene}

To confirm that disruption of the $\mathrm{SO}_{-} 1860$ gene was responsible for the distinct morphotype and enhanced current generation by strain EC-2, an in-frame deletion mutant of SO_1860 (designated $\Delta S_{-}{ }_{1} 1860$ ) was constructed. When cultivated on LB plates, $\Delta$ SO_1860 displayed a slightly rough colony morphotype as compared with WT (Figure 1). However, colonies formed by $\Delta \mathrm{SO}_{-} 1860$ were similar in size to those of WT, and smaller than those of strain EC-2. As colony morphology is influenced by cell surface structures and physicochemical properties [32-34], we next evaluated cell surface hydrophobicity of WT, EC-2, and $\Delta$ SO_1860 by measuring the affinity of cells to hexadecane (Figure 4). The EC-2 and $\Delta S O$ _1860 mutants were more hydrophobic than WT, but the hydrophobicity of $\Delta \mathrm{SO}_{1} 1860$ was lower than that of EC-2. These results were consistent with the rough colony appearance of both mutant strains.

Current generation by $\Delta \mathrm{SO} \_1860$ was also analyzed and compared with that of WT and strain EC-2 (Figure 5) using a small-volume, single-chamber EC reactor equipped with a working electrode poised at $+0.2 \mathrm{~V}$ ( $v s . \mathrm{Ag} / \mathrm{AgCl}$ ) for the stable and short-term measurement of electric current

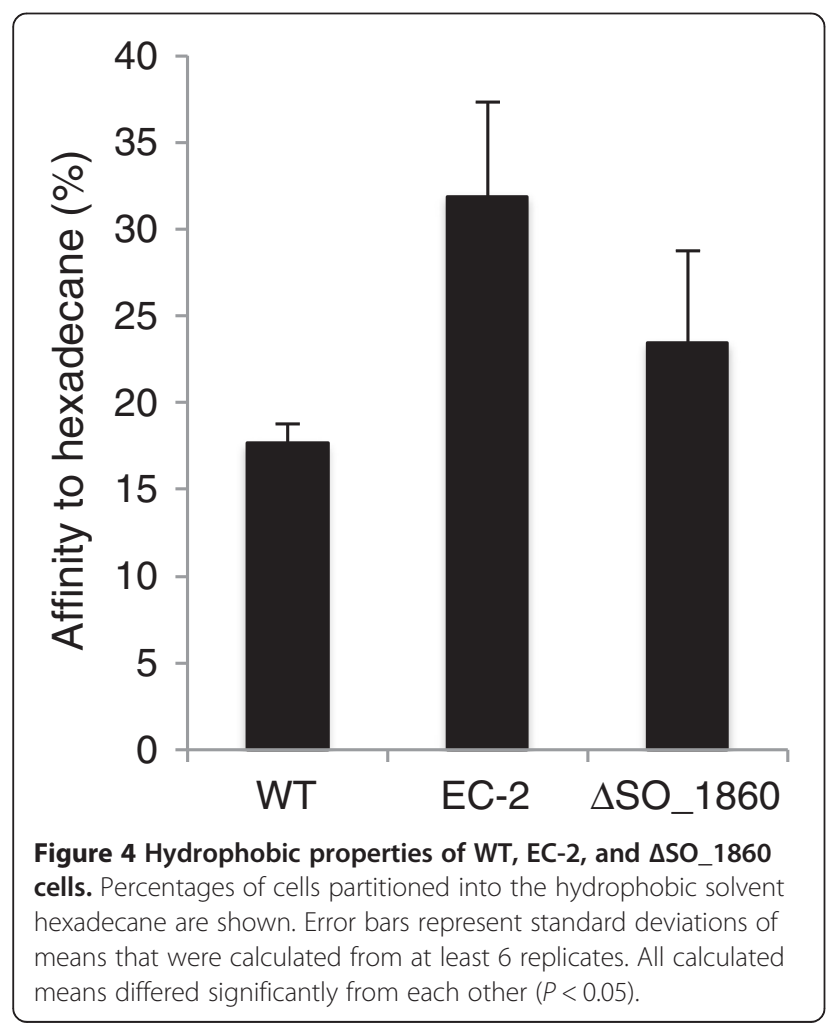

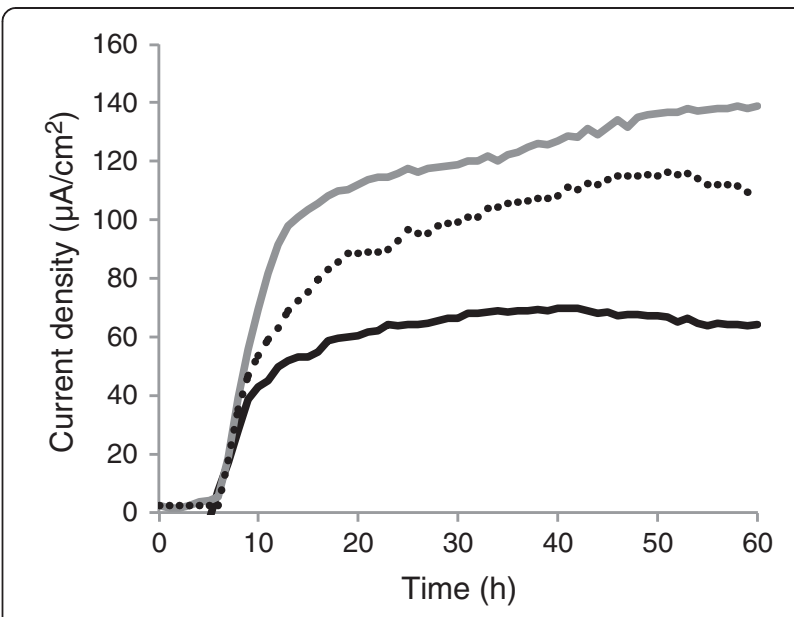

Figure 5 Comparison of current generation by WT (black line), EC-2 (grey line), and $\Delta$ SO_1860 (dotted line) in ECs. Results represent means of at least two parallel but independent experiments.

(Figure 5). Strain EC-2 and $\Delta$ SO_1860 generated 90\% and $60 \%$, respectively, higher current than WT. These results indicated that although the disruption of SO_1860 affected colony morphology, cell surface hydrophobicity, and current-generating ability, it was not the only cause for the distinct phenotype of strain EC-2. The reason for this will be discussed later.

\section{Expression of CPS biosynthesis genes}

Our previous study found that CPSs negatively affect current generation in S. oneidensis MR-1 [25]. In addition, the CPS biosynthesis genes, which are located within a large gene cluster (SO_3193 to SO_3171), are reported to be down-regulated in a $S O \_1860$ knockout mutant [30]. We therefore hypothesized that CPS biosynthesis was repressed in strain EC-2, resulting in the increased ability of this mutant to generate current. To examine this hypothesis, WT, EC-2 and $\Delta$ SO_1860 cells were grown in LMM under fumarate-reducing conditions, and expression levels of three CPS biosynthesis genes (SO_3172, SO_3177, and SO_3179) were determined by the quantitative reverse transcription PCR (qRT-PCR) analysis of extracted total RNA (Figure 6). In both EC-2 and $\triangle \mathrm{SO} \_1860$, expression levels of the CPS biosynthesis genes were decreased to $40 \%-54 \%$ of those in WT $\left(\log _{2}\right.$-transformed fold changes $\left[\log _{2} \mathrm{FC}\right]<-0.89$ ), supporting the speculation that the repression of CPS is related to the increased current generation by these two mutant strains. However, the expression levels of SO_3172 and SO_3179 did not significantly differ between EC-2 and $\triangle S O$ S1860, and the expression level of $S O \_3177$ was slightly decreased in $\triangle S O \_1860$ as compared with that in EC-2. These results suggest that the high current-generating capability of EC-2, which exceeded that of $\Delta S O$ _ 1860, was not simply due to the decreased expression of CPS biosynthesis genes. 


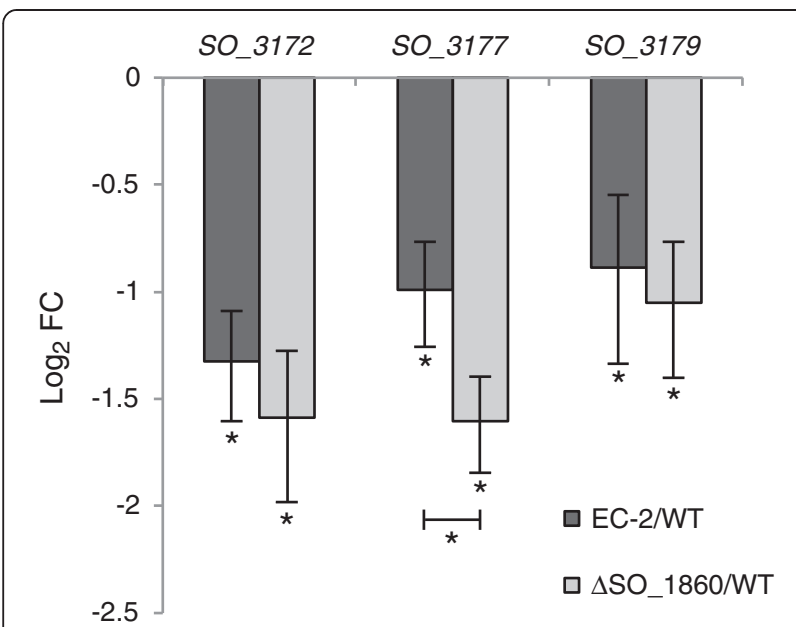

Figure 6 qRT-PCR analyses of CPS biosynthesis genes in WT, EC-2, and $\Delta$ SO_ 1860 cells. Results are expressed as $\log _{2}$-fold changes $(F C)$ relative to expression levels in WT cells. The error bars represent the standard deviation calculated from at least four measurements. Asterisks indicate a statistically significant difference $(P<0.05)$ between expression levels in the three strains.

\section{Differentially expressed genes in EC-2}

To investigate why EC-2 exhibited a distinct phenotype from $\Delta S O$ _ 1860 , gene expression profiles in the two mutants were analyzed and compared by transcriptome analysis. Total RNA from EC-2 and $\Delta$ SO_1860 was prepared from cells cultured in LMM under fumaratereducing conditions and subjected to microarray analysis. The reliability of the microarray analysis was validated by qRT-PCR of five selected genes (Additional file 4: Figure S4). A high correlation $\left(r^{2}=0.97\right)$ was observed between the microarray and qRT-PCR results.

Microarray analysis revealed that 26 genes had significantly different expression levels $\left(P<0.05, \mid \log _{2}\right.$ $\mathrm{FC} \mid \geq 1.0$ ) in strains EC-2 and $\Delta \mathrm{SO}_{-} 1860$ (Table 1). Among the 26 genes, 11 genes were up-regulated and 15 genes were down-regulated in strain EC-2 as compared with $\triangle \mathrm{SO}_{-} 1860$. The up-regulated genes included those assigned to the COG (Clusters of Orthologous Groups of proteins [35]) categories of "Transcription", "Signal transduction mechanisms", "Nucleotide transport and metabolism", and "Amino acid transport and metabolism". Notably, expression levels of the genes involved in methionine biosynthesis, metR and metE, were remarkably up-regulated in EC-2. It is known that MetR acts as a transcriptional activator for the metE gene [36,37], suggesting that the overexpression of metE is related to that of metR. The expression of luxS, encoding S-ribosyl homocysteinase, was also up-regulated in EC-2. This is notable because LuxS is involved in biofilm development in many bacteria, including E. coli and S. oneidensis MR-1 $[38,39]$. In addition, it was found that the $S O \_1860$ gene was up-regulated in EC-2 compared to $\Delta \mathrm{SO} \_1860$, but this was due to the complete deletion of this gene in $\Delta \mathrm{SO}_{-} 1860$.

The expression levels of metR, metE, and $\operatorname{luxS}$ were also examined and compared in WT, EC-2, and $\Delta \mathrm{SO} \_1860$ by qRT-PCR (Figure 7). It was confirmed that these three genes were overexpressed in EC-2, whereas their expression levels were not significantly different between WT and $\Delta \mathrm{SO}_{-} 1860$, suggesting that the overexpression of these genes is attributable to mutations specific to EC-2.

The down-regulated genes in EC-2 (Table 1) included a number of genes located within the LambdaSo prophage region (SO_2939 to SO_3013) [40]. The transcriptome analysis also revealed that the $S_{-} O_{1} 1861$ gene, which is located immediately downstream of $S O \_1860$, was also down-regulated in EC-2. This was considered to be due to a polar effect caused by insertion of the Tn into $S_{-}{ }_{1} 1860$. The SO_0351, SO_2005, and SO_4564 genes, which are categorized into the COG category "Signal transduction mechanisms" and "Cell wall/membrane/envelope biogenesis", respectively, were also down-regulated in EC-2.

\section{Discussion}

In this study, we isolated and characterized the novel $S$. oneidensis MR-1 mutant strain EC-2, which exhibits an increased ability to generate current in MFC and EC reactors. In addition, strain EC-2 forms flat, rough, and extraordinarily large colonies that are clearly distinct from the morphology of WT colonies (Figure 1), implying that cell surface structure and physicochemical properties are altered in this mutant. As we previously provided evidence that cell surface hydrophobicity influences the adhesiveness of $S$. oneidensis cells to graphite felt electrodes and affects current generation in MFCs [25], we also examined the adhesiveness and hydrophobicity of EC-2 cells. Compared to WT, EC-2 cells attached with higher frequency to graphite felt anodes (Figure 3) and had a more hydrophobic surface (Figure 4). Taken together, these results suggest that mutations introduced into EC-2 modified the cell surface structure and hydrophobicity, resulting in the enhanced adhesiveness of cells to graphite felt electrodes and increased current generation. A similar trend has also been observed for strain $\triangle \mathrm{SO}_{2} 3177$, which contains a mutation in a gene necessary for CPS synthesis and shows altered cellsurface hydrophobicity and enhanced the adhesiveness to graphite felt anodes [25]. We therefore hypothesized that genes involved in the synthesis of CPS or other cell surface structures were mutated in strain EC-2.

We determined that the SO_1860 (uvrY) gene, which encodes a DNA-binding response regulator of the BarA/ UvrY two-component regulatory system, was disrupted in strain EC-2 by Tn insertion. In S. oneidensis MR-1, SO_1860 is involved in the transcriptional regulation of 
Table 1 Differentially expressed genes in strain EC-2 $\left(P<0.05,\left|\log _{2} \mathrm{FC}\right| \geq 1.0\right)$

\begin{tabular}{|c|c|c|c|c|}
\hline Locus tag & Gene & Putative function & COG description ${ }^{1}$ & $\log _{2} F C^{2}$ \\
\hline \multicolumn{5}{|c|}{ Up-regulated genes } \\
\hline SO_0817 & metR & Transcriptional activator protein MetR & Transcription & 3.33 \\
\hline SO_0818 & metE & $\begin{array}{l}\text { 5-Methyltetrahydropteroyltriglutamate-homocysteine } \\
\text { methyltransferase }\end{array}$ & Amino acid transport and metabolism & 6.49 \\
\hline SO_1101 & luxS & S-Ribosylhomocysteinase & Signal transduction mechanisms & 1.65 \\
\hline SO_1860 & - & Response regulator & $\begin{array}{l}\text { Signal transduction mechanisms, } \\
\text { Transcription }\end{array}$ & 5.97 \\
\hline SO_2404 & $\operatorname{aroA}$ & 3-Phosphoshikimate 1-carboxyvinyltransferase & Amino acid transport and metabolism & 1.17 \\
\hline SO_3471 & glyA & Serine hydroxymethyltransferase & Amino acid transport and metabolism & 1.55 \\
\hline SO_3534 & murJ & Peptidoglycan lipid II flippase & General function prediction only & 1.17 \\
\hline SO_4189 & - & Hypothetical protein SO_4189 & Carbohydrate transport and metabolism & 1.04 \\
\hline SO_4233 & leud & Isopropylmalate isomerase small subunit & Amino acid transport and metabolism & 1.69 \\
\hline SO_4596 & - & Copper-transporting ATPase domain-containing protein & - & 2.09 \\
\hline SO_4731 & add & Adenosine deaminase & Nucleotide transport and metabolism & 1.42 \\
\hline \multicolumn{5}{|c|}{ Down-regulated genes } \\
\hline SO_0351 & - & LuxR family DNA-binding response regulator & $\begin{array}{l}\text { Signal transduction mechanisms, } \\
\text { Transcription }\end{array}$ & -1.04 \\
\hline SO_1861 & uvrC & Excinuclease $A B C$ subunit $C$ & Replication, recombination and repair & -1.30 \\
\hline SO_2005 & - & DksA-type zinc finger protein & Signal transduction mechanisms & -1.16 \\
\hline SO_2906 & - & Hypothetical protein SO_2906 & Inorganic ion transport and metabolism & -1.92 \\
\hline SO_2945 & - & Lambda phage tail fiber protein & - & -1.45 \\
\hline SO_2946 & - & Lambda phage protein with carbohydrate-binding module & - & -1.10 \\
\hline SO_2953 & $\mathrm{H}$ & Prophage LambdaSo, tail length tape meausure protein & Function unknown & -1.48 \\
\hline SO_2954 & - & Hypothetical protein SO_2954 & - & -1.53 \\
\hline SO_2955 & - & Lambda phage minor tail protein $\mathrm{G}$ & - & -1.62 \\
\hline SO_2956 & - & Prophage LambdaSo, major tail protein $\mathrm{V}$, putative & - & -1.60 \\
\hline SO_2957 & - & Lambda phage protein of unknown function & - & -1.35 \\
\hline SO_2965 & - & Prophage LambdaSo, HK97 family portal protein & Function unknown & -1.24 \\
\hline SO_2968 & - & Phage terminase small subunit & Replication, recombination and repair & -1.03 \\
\hline SO_2969 & - & Prophage LambdaSo, holin, putative & Defense mechanisms & -1.24 \\
\hline SO_4564 & - & TonB2 protein, putative & Cell wall/membrane/envelope biogenesis & -1.15 \\
\hline
\end{tabular}

${ }^{1}$ Functional categorization by COG.

${ }^{2}$ Log $_{2}$-transformed fold change (EC-2/ASO_1860).

a large number of genes, including CPS biosynthesis genes [30], suggesting that disruption of $\mathrm{SO}_{-} 1860$ in strain EC-2 modified cell surface structure and led to the enhanced current generation in MFCs. The involvement of SO_1860 in current generation was examined by constructing an in-frame deletion mutant of $\mathrm{SO}_{-} 1860$ ( $\left.\triangle \mathrm{SO} \_1860\right)$, which was found to generate higher current in an EC, indicating the involvement of SO_1860 in current generation. $\triangle \mathrm{SO} \_1860$ cells also exhibited altered colony morphology and increased cell surface hydrophobicity (Figure 4), and qRT-PCR analyses demonstrated that the expression of several CPS biosynthesis genes (SO_3172, SO_3177, and SO_3179) was repressed (Figure 6). These results support the notion that disruption of the SO_1860 gene affects CPS synthesis and cell surface hydrophobicity, resulting in increased current generation. However, strain EC-2 formed larger colonies, exhibited higher hydrophobicity, and generated higher current than $\Delta \mathrm{SO}_{-} 1860$, indicating that the disruption of $S_{-} O_{1860}$ was not the only cause for the distinct phenotype of strain EC-2. As Southernblotting analysis confirmed that EC-2 had a single Tn insertion in SO_1860 (data not shown), it is likely that unknown mutations, in addition to the Tn insertion, may have been spontaneously introduced during the long-term (approximately 40 days) electrochemical cultivation of strain EC-2. Another explanation is that gene(s) located downstream of the Tn insertion site in SO_1860 are 


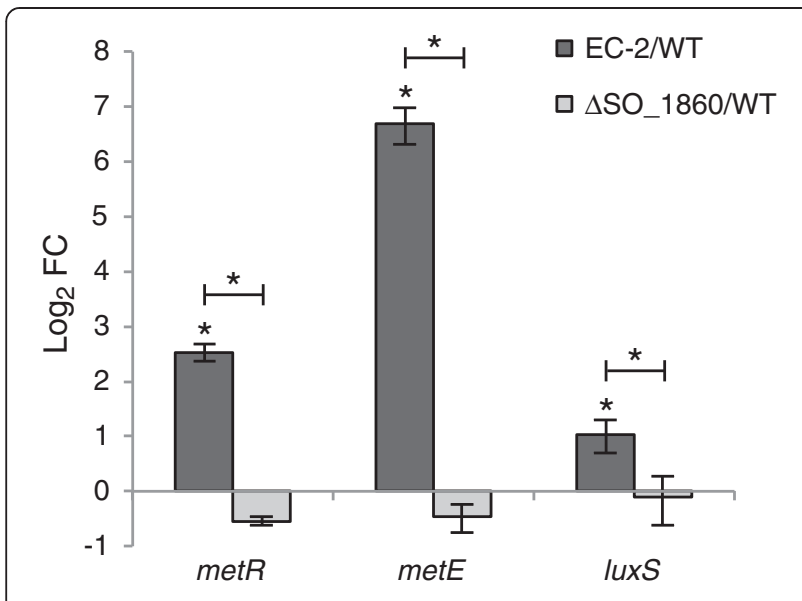

Figure 7 qRT-PCR analyses of the metR, metE, and luxS genes in WT, EC-2, and $\Delta$ SO_1860 cells. Results are expressed as $\log _{2}$-fold changes (FC) relative to expression levels in WT cells. The error bars represent the standard deviation calculated from at least four measurements. Asterisks indicate a statistically significant difference $(P<0.05)$ between expression levels in the three strains.

differently expressed in EC-2. However, it is unlikely that the distinct phenotypic features of EC-2 are attributable to the decreased expression of SO_1861 (the excinuclease $A B C$ subunit $C$ gene; Table 1). Further investigation, such as genome sequencing of strain EC-2, is needed to examine these hypotheses.

We examined and compared the gene expression profiles of EC-2 and $\Delta$ SO_1860 to understand the physiological differences between these two strains. qRT-PCR (Figure 6) and microarray (Table 1) analyses demonstrated that expression of CPS biosynthesis genes did not largely differ between EC-2 and $\triangle$ SO_1860, suggesting that the distinct features of EC-2 are not attributable to differential expression of CPS synthesis genes. However, we identified a number of genes that were differentially expressed between EC-2 and $\Delta$ SO_1860 (Table 1). Notably, the methionine biosynthesis genes $m e t R$ and metE were highly up-regulated (10- and 90-fold, respectively) in EC-2, although it remains unclear why these genes were overexpressed. It is also interesting that the $\operatorname{lux} S$ gene was upregulated in $\mathrm{EC}-2$, as this enzyme catalyzes the conversion of S-ribosyl homocysteine to homocysteine and 4,5-dihydroxy-2,3-pentanedione (DPD), and is widely conserved in both Gram-negative and Gram-positive bacteria [41-43]. LuxS functions as an autoinducer-2 (AI-2) synthase because DPD is spontaneously converted to AI-2 [42-44]. LuxS is also involved in the activated methyl cycle (AMC) $[38,43]$ which is responsible for the synthesis of homocysteine, methionine, and S-adenosylmethionine (SAM), a major methyl donor source that is utilized for various cellular processes including the methylation of DNA and methyl-accepting chemotaxis proteins [43,45]. MetE (methionine synthase) and MetR (transcriptional activator for met genes) are also involved in the AMC. In S. oneidensis, disruption of $\operatorname{lux} S$ negatively affects biofilm development on solid surfaces by interrupting the AMC [39]. It has been also reported that a luxS-complemented (overexpressing) mutant of $E$. coli was deficient in pili production and formed a thicker biofilm than the WT strain, phenotypes that were suggested to be due to the depletion of SAM resulting from elevated luxS expression [38]. In addition, a metR mutant ( $\Delta$ metR) of Pseudomonas aeruginosa exhibited altered colony morphology due to a severe defect in swarming motility [46], suggesting that the AMC is associated with cell motility and colony morphology. It is therefore possible that the overexpression of AMCassociated genes in EC-2 influences cell surface structure or motility, resulting in the altered colony morphology and increased adhesiveness of cells to electrodes. Studies are underway to investigate this possibility.

The function and expression of the metR, metE, and luxS genes are considered to be closely related, because homocysteine, one of the products of LuxS, acts as a coregulator for MetR and stimulates the transcription of $m e t E$ [47]. We found a potential MetR-binding site in the upstream intergenic region of the MR-1 luxS gene (5'TGAGATGATTTCA-3') that closely matches the consensus MetR-binding sequence reported in E. coli and other bacteria (5'-TGAANNANNTTCA-3') [48]. A similar sequence was also identified in the intergenic region between $m e t E$ and $m e t R$ (5'-TGAGCGAAATTCA-3'). These findings suggest the possibility that MetR regulates the expression of luxS, as well as that of met genes, in S. oneidensis MR-1. A putative MetR-binding sequence was also found upstream of glyA (5'-TGAGGTGCATTCA-3'). Because MetR activates the transcription of $g l y A$ in $E$. coli [49], it is likely that the overexpression of metR in EC-2 resulted in the increased expression of $g l y A$. In addition to these AMC-related genes, the microarray analysis detected 20 genes other than $S_{-}{ }_{1} 1860$ and $S_{-}{ }_{1} 1861$ that were differentially regulated in EC-2, including 10 genes located within the LambdaSo prophage region (Table 1). Although it has been reported that prophage-mediated cell lysis enhances biofilm formation in S. oneidensis MR-1 [40], the regulation and involvement of these genes in the observed phenotype of EC-2 is unknown.

\section{Conclusions}

The present study indicates that SO_1860 (uvrY) and other biofilm formation-related genes, including those involved in CPS biosynthesis and the AMC, play important roles in determining colony morphology, cell surface properties, and current-generating capability of $S$. oneidensis MR-1. Thus, it might be possible to control the adhesion of cells to electrodes by altering the expression of these genes, and thereby increase the efficiency of BESs. Although the present study focused on the altered 
gene expression profiles of strain EC-2, proteomic and metabolomic approaches will also provide useful information for understanding the distinct features of this mutant. Future studies will be conducted to elucidate the mechanisms underlying the increased currentgenerating capability of strain EC-2.

\section{Methods}

\section{Bacterial strains and plasmids}

S. oneidensis MR-1 was obtained from American Type Culture Collection (ATCC). E. coli strains [8] were routinely cultured in Luria-Bertani (LB) medium at $37^{\circ} \mathrm{C}$. The E. coli mating strain (WM6026) required supplementation of the medium with $100 \mu \mathrm{g} \mathrm{ml}^{-1}$ 2,6-diaminopimelic acid (DAP) for growth. Shewanella strains were cultured at $30^{\circ} \mathrm{C}$ in either LB or lactate minimal medium [25] supplemented with $0.2 \mathrm{~g} \mathrm{liter}^{-1}$ casamino acids and $10 \mathrm{ml}$ liter $^{-1}$ each of amino acid and trace mineral solutions (LMM). When necessary, $50 \mu \mathrm{g} \mathrm{ml}^{-1}$ kanamycin $(\mathrm{Km})$ was added to culture media. Agar plates contained 1.6\% Bacto agar (Difco).

\section{Construction of a mutant library}

Random Tn mutagenesis of S. oneidensis MR-1 was performed by filter mating with $E$. coli WM6026 harboring the suicide plasmid pBSL180 [50], which contained miniTn $10 \mathrm{Km}^{\mathrm{r}}$, according to a previously described method $[25,26]$. After transformed cells were grown on LB-agar plates containing DAP at $30^{\circ} \mathrm{C}$ for $8 \mathrm{~h}$, the cells were washed in $10 \mathrm{mM} \mathrm{MgSO}_{4}$ and then aerobically grown in LMM containing $\mathrm{Km}$ for $24 \mathrm{~h}$.

\section{Selection of mutants}

Selection of mutants from the random Tn-insertion library was carried out using a previously described method [26] with slight modifications. Briefly, a cylindrical electrochemical cell (EC; $500 \mathrm{ml}$ capacity) equipped with a graphite-felt working electrode (WE; $50 \mathrm{~cm}^{2}$; GF-80-3 F, Sohgoh Carbon), air diffusion-type counter electrode (approximately $20 \mathrm{~cm}^{2}$; $0.7 \mathrm{mg}$ platinum $/ \mathrm{cm}^{2}$; and four polytetrafluoroethylene layers) was constructed as described elsewhere [51], and $\mathrm{Ag} / \mathrm{AgCl}$ was used as the reference electrode (HX-R5, Hokuto Denko). The EC was filled with $450 \mathrm{ml}$ LMM, purged with pure nitrogen gas, and then inoculated with the mutant library at a final cell concentration of approximately $2 \times 10^{6} \mathrm{ml}^{-1}$. The EC was incubated at $30^{\circ} \mathrm{C}$ for 40 days under constant agitation, and the working electrode was poised at $+0.2 \mathrm{~V}$ vs. the $\mathrm{Ag} / \mathrm{AgCl}$ reference electrode using a potentiostat (Multipotentiostat 2092, Toho Giken). Current was monitored using the potentiostat, and current density $\left(\mathrm{A} \mathrm{cm}^{-2}\right)$ was calculated based on the anode projection area $\left(50 \mathrm{~cm}^{2}\right)$. When the current density fell below $0.1 \mathrm{~mA}$, lactate was injected into the $\mathrm{EC}$ at a final concentration of $10 \mathrm{mM}$.

\section{Isolation of mutants}

After the 40-day electrochemical selection in the EC, the electrolyte was collected, serially diluted, and spread on agar plates containing LB supplemented with $\mathrm{Km}$. Colonies that formed on the agar plates were randomly selected and purified by re-streaking for isolated colonies. Isolated mutants were grown in LB medium supplemented with $\mathrm{Km}$ and stored at $-80^{\circ} \mathrm{C}$ in $15 \%$ (v/v) glycerol. Tn-insertion sites of the isolated mutants were identified according to a method described previously [26].

\section{Gene disruption}

In-frame disruption of the SO_1860 gene in strain MR-1 was performed using a two-step homologous recombination method with suicide plasmid pSMV-10, as described previously $[8,25,52]$. Briefly, a $1.6-\mathrm{kb}$ fusion product, consisting of an upstream (768 bp) and downstream (798 bp) sequence of the $S O \_1860$ gene (768 bp) joined by an 18-bp linker sequence, was constructed by PCR and in-vitro extension using the primers listed in Additional file 5: Table S1. The amplified fusion product was ligated into the SpeI site of pSMV10, generating pSMV-1860, which was then introduced into MR-1 by filter mating with $E$. coli WM6026. Transconjugants (single-crossover clones) were selected on LB plates containing $\mathrm{Km}$ and further cultivated for $20 \mathrm{~h}$ in LB medium lacking antibiotics. The cultures were then spread onto LB plates containing $10 \%(\mathrm{w} / \mathrm{v})$ sucrose to isolate $\mathrm{Km}$ sensitive double-crossover mutants. Disruption of the SO_1860 gene in the obtained strains was confirmed by PCR. One representative mutant strain in which the SO_1860 gene was disrupted in-frame was selected and designated $\Delta \mathrm{SO} \_1860$.

\section{Evaluation of mutants in MFCs and ECs}

In MFC experiments, microbial current generation was measured using a single-chamber MFC equipped with a graphite felt anode $\left(50 \mathrm{~cm}^{2} ; \mathrm{GF}-80-3 \mathrm{~F}\right)$ and air cathode (approximately $20 \mathrm{~cm}^{2} ; 0.7 \mathrm{mg}$ platinum $/ \mathrm{cm}^{2}$; and four polytetrafluoroethylene layers), as previously described [25]. Bacterial cells were inoculated into the MFC chamber, which contained $450 \mathrm{ml}$ LMM supplemented with $10 \mathrm{mM}$ lactate, at an initial optical density at $600 \mathrm{~nm}$ $\left(\mathrm{OD}_{600}\right)$ of 0.005 . Upon depletion of lactate, a $4.5 \mathrm{M}$ stock solution of lactate was injected into the reactor to increase the concentration of lactate to $10 \mathrm{mM}$. The anode and cathode were connected via electric wires and an external resistor $(100 \Omega)$, and the voltage across the resistor was measured using a voltage data logger (HA-1510, Graphtec). Current $(I[\mathrm{~A}])$ was calculated using the equation: $I=$ $E / R$, where $\mathrm{E}[\mathrm{V}]$ is the cell voltage and $\mathrm{R}[\Omega]$ is the resistance. Current density $\left(\mathrm{A} \mathrm{cm}^{-2}\right)$ was calculated using the anode projection area $\left(50 \mathrm{~cm}^{2}\right)$. A polarization curve was generated using a potentiostat (HSV-100; Hokuto Denko), 
from which the maximum power density $\left(P_{\max }\left[\mathrm{W} \mathrm{cm}{ }^{-2}\right]\right)$ and short-circuit current $\left(I_{\mathrm{sc}}\left[\mathrm{A} \mathrm{cm}^{-2}\right]\right)$ were obtained as described elsewhere [53]. Reproducibility was examined in at least three independent measurements, and typical data are shown here. The protein contents in planktonic cells, anode biofilms, and cathode biofilms in MFC reactors were determined using a BCA protein assay Kit (Pierce) according to a method described previously [25].

A low-volume (18 ml capacity), single-chamber EC equipped with a graphite felt working electrode $\left(2.3 \mathrm{~cm}^{2}\right.$; poised at $+0.2 \mathrm{~V} v s$. an $\mathrm{Ag} / \mathrm{AgCl}$ reference electrode [HXR5, Hokuto Denko]) was used to monitor and compare current generation by WT, EC-2, and $\Delta \mathrm{SO} \_1860$ cells. A platinum wire ( $5 \mathrm{~cm}, \phi 0.3 \mathrm{~mm}$; Nilaco) was used as the counter electrode. Bacterial cells were inoculated into the EC chamber, which containing $15 \mathrm{ml}$ LMM supplemented with $10 \mathrm{mM}$ lactate, at an initial $\mathrm{OD}_{600}$ of 0.01 . Current was monitored using a potentiostat (HA-1510; Hokuto Denko), and current density $\left(\mathrm{A} \mathrm{cm}^{-2}\right)$ was calculated based on the anode projection area $\left(2.3 \mathrm{~cm}^{2}\right)$. Reproducibility was examined in at least three independent measurements.

\section{Hydrophobicity assay}

Cell hydrophobicity was analyzed by the bacterial adhesion to hydrocarbon method (BATH), as described previously $[25,54]$. Briefly, Shewanella cells were suspended in $2.4 \mathrm{ml}$ of $0.15 \mathrm{M} \mathrm{NaCl}$ at an $\mathrm{OD}_{600}$ of 0.3 (approximately $3 \times 10^{8} \mathrm{CFU} \mathrm{ml} \mathrm{m}^{-1}$ ) and vortexed for $60 \mathrm{~s}$ in the presence of $0.4 \mathrm{ml}$ hexadecane. The mixture was allowed to stand for $15 \mathrm{~min}$ at room temperature to ensure that the two phases were completely separated before a 1-ml sample was removed from the aqueous phase for measuring the $\mathrm{OD}_{600}$. The percentage of cells transferred to the hexadecane phase was subsequently calculated using the equation: affinity $(\%)=100 \times\left[1-\left(A / A_{0}\right)\right]$, where $A_{0}$ is the $\mathrm{OD}_{600}$ of the bacterial suspension before mixing with hexadecane and $\mathrm{A}$ is the $\mathrm{OD}_{600}$ after mixing. All measurements were performed in at least six replicates, and data were statistically analyzed by one-way analysis of variance (ANOVA) in combination with Holm's multiple-comparison test using js-STAR software (http://www.kisnet.or.jp/nappa/ software/star/). A $P$-value of 0.05 was considered statistically significant.

\section{RNA extraction}

Shewanella cells were grown anaerobically in LMM (containing $15 \mathrm{mM}$ lactate) supplemented with $20 \mathrm{mM}$ fumarate as the electron acceptor, and cells were harvested at the early stationary growth phase $\left(\mathrm{OD}_{600}\right.$ of 0.16-0.18). Total RNA was extracted using Trizol reagent (Invitrogen) following the manufacturer's instructions and subsequently purified using an RNeasy Mini Kit and RNase-Free DNase Set (Qiagen). The quality of extracted RNA was evaluated using an Agilent 2100
Bioanalyzer with RNA 6000 Pico reagents and RNA Pico Chips (Agilent Technologies) according to the manufacturer's instructions. The purified RNA was then used for qRT-PCR and microarray transcriptome analysis.

\section{qRT-PCR}

qRT-PCR was performed according to a method described previously $[55,56]$ with slight modifications. RT and subsequent quantitative PCR were conducted using a LightCycler 1.5 instrument (Roche) following the manufacturer's instructions. The PCR mixture $(20 \mu \mathrm{l})$ contained $1 \mu \mathrm{l}$ diluted RNA (150 ng for luxS, and $15 \mathrm{ng}$ for other genes), $1.3 \mu \mathrm{l}$ of $50 \mathrm{mM} \mathrm{Mn}(\mathrm{OAc})_{2}$ solution, $7.5 \mu \mathrm{l}$ LightCycler RNA Master SYBR Green I (Roche), and $0.15 \mu \mathrm{M}$ of the primers listed in Supplemental Table 1. A standard curve was drawn using serial dilutions of PCR fragments of each gene. Specificity of the quantitative PCR was verified by dissociation-curve analysis. The expression levels of the target genes were normalized based on the expression level of the $16 \mathrm{~S}$ rRNA gene. All measurements were performed in quadruplicate at a minimum, and data were statistically analyzed by one-way ANOVA with Bonferroni's multiple-comparison test using SAS software (version 9.3, SAS Institute). A P-value of 0.05 was considered statistically significant.

\section{Microarray analysis}

A custom 60-mer oligonucleotide microarray $(8 \times 15 \mathrm{~K}$; Agilent Technologies) containing (maximally) three different probes per gene was designed using the annotated chromosomal and megaplasimd sequences of $S$. oneidensis MR-1 (Genbank accession nos. AE014299 and AE014300, respectively). Specific oligonucleotide probes were designed for 4,772 genes (corresponding to $96.6 \%$ of the total annotated genes) using the eArray protocol (Agilent Technologies) and fabricated on slide glass by SurePrint technology (Agilent Technologies). Fluorescence labeling of cDNA, and hybridization and scanning of hybridized arrays were performed according to the manufacturer's protocols for gene expression arrays for prokaryotes (Agilent One-Color Microarray-Based Prokaryote Analysis, version 1.4, http:// www.chem.agilent.com). Briefly, cyanine 3 (Cy3)-labeled cDNA was synthesized from $5 \mu \mathrm{g}$ total RNA using the FairPlay III Microarray Labeling KitStratagene with CyDye Cy3 mono-Reactive Dye (GE Healthcare). The labeled cDNA was quantified using a NanoDrop ND-1000 Spectrophotometer (Thermo Scientific). For each array, $20 \mu \mathrm{l}$ of the purified Cy3-labeled cDNA $(20 \mathrm{ng} / \mu \mathrm{l})$ was mixed with $5 \mu \mathrm{l}$ of $10 \times$ Blocking Agent and $25 \mu \mathrm{l}$ of $2 \times$ GEx Hybridization Buffer HI-RPM. The resultant mixture $(40 \mu \mathrm{l})$ was hybridized to the array at $65^{\circ} \mathrm{C}$ for $17 \mathrm{~h}$. After hybridization, each slide was washed with Gene Expression Wash Buffer I (Agilent Technologies) at room temperature for $1 \mathrm{~min}$, followed by Gene Expression 
Wash Buffer II (Agilent Technologies) at $37^{\circ} \mathrm{C}$ for $1 \mathrm{~min}$. Slides were air dried and then scanned using an Agilent DNA Microarray Scanner at 5- $\mu \mathrm{m}$ resolution. Data acquisition was performed using the Feature Extraction Software version 8.1 (Agilent Technologies). Gene expression data ( $\mathrm{n}=3$ biological replicates) were normalized and statistically analyzed using GeneSpring GX version 11.5 (Agilent Technologies). The unpaired Student's t-test and the Benjamini-Hochberg false discovery rate correction were used for statistical analysis. Differential expression for each probe was considered statistically significant when the fold change (FC) was $\geq 2.0$ or $\leq 0.5\left(\left|\log _{2} \mathrm{FC}\right| \geq 1.0\right)$ at a $P$-value of $<0.05$. The average FC was calculated for each gene from the values of the probes with altered expression. The microarray data have been deposited in the NCBI Gene Expression Omnibus (GEO) under the accession number GSE50443.

\section{Additional files}

Additional file 1: Figure S1. Current generation in an EC inoculated with a random transposon mutant library of S. oneidensis MR-1.

Additional file 2: Figure S2. Colonies of mutants with altered morphology isolated after electrochemical enrichment. Mutants with distinct colony morphology were picked and further cultivated on a LB agar plate for 2 days. An arrowhead indicates a colony similar in size to that of WT.

Additional file 3: Figure S3. Current generation by strain EC-2 (grey line) and WT (black line) in single-chamber MFCs. An arrowhead indicates the time point at which polarization (Figure 2A) and power (Figure 2B) curves were measured. Reproducibility was examined in at least three independent operations, and typical data are shown.

Additional file 4: Figure S4. Comparison of transcriptional changes in strains EC-2 and $\Delta$ SO_1860 as determined by microarray and qRT-PCR analyses. $\mathrm{Log}_{2}$-transformed fold changes $\left(\mathrm{Log}_{2} \mathrm{FC}\right)$ in the expression levels (EC-2/ASO_1860) of 5 selected genes determined by microarray analysis were plotted against the values determined by qRT-PCR.

Additional file 5: Table S1. Primers used in this study.

\section{Abbreviations}

EET: Extracellular electron transfer; CPS: Cell-surface polysaccharide; EC: Electrochemical cell; BES: Bioelectrochemical system; MFC: Microbial fuel cell; OM-cyt: Outer-membrane cytochrome; Tn: Transposon; LB: Luria-Bertani; DAP: 2:6-Diaminopimelic acid; LMM: Lactate minimal medium; WT: Wild-type; DPD: 4,5-Dihydroxy-2,3-pentanedione; FC: Fold change; AMC: Activated methyl cycle; SAM: S-adenosylmethionine.

\section{Competing interests}

The authors declare that they have no competing interests.

\section{Authors' contributions}

AK participated in the design of the study, performed the molecular genetic studies, and drafted the manuscript. $\mathrm{HO}$ participated in the evaluation of mutants, qRT-PCR, and microarray analysis. NT participated in the isolation of mutants. KH participated in the design and coordination of the study. KW conceived of the study, participated in its design and coordination, and performed manuscript editing. All authors read and approved the final manuscript.

\section{Acknowledgements}

This work was supported by the Exploratory Research for Advanced Technology (ERATO) program of the Japanese Science and Technology Agency (JST). We thank Reiko Hirano and Ayako Matsuzawa for technical assistance.

\section{Author details}

${ }^{1}$ School of Life Sciences, Tokyo University of Pharmacy and Life Sciences, 1432-1 Horinouchi, Hachioji 192-0392, Tokyo, Japan. ²Department of Applied Chemistry, The University of Tokyo, 7-3-1 Hongo, Bunkyo-ku 113-8656, Tokyo, Japan. ${ }^{3}$ Hashimoto Light Energy Conversion Project, ERATO/JST, The University of Tokyo, 7-3-1 Hongo, Bunkyo-ku 113-8656, Tokyo, Japan. ${ }^{4}$ Present address: Advanced Technologies Research Laboratories, Idemitsu Kosan, 1200 Kamiizumi, Sodegaura 299-0293, Chiba, Japan.

Received: 15 April 2014 Accepted: 10 July 2014

Published: 16 July 2014

\section{References}

1. Fredrickson JK, Romine MF, Beliaev AS, Auchtung JM, Driscoll ME, Gardner TS, Nealson KH, Osterman AL, Pinchuk G, Reed JL, Rodionov DA, Rodrigues JLM, Saffarini DA, Serres MH, Spormann AM, Zhulin IB, Tiedje JM: Towards environmental systems biology of Shewanella. Nat Rev Microbiol 2008, 6:592-603.

2. Nealson $\mathrm{KH}$, Saffarini D: Iron and manganese in anaerobic respiration: environmental significance, physiology, and regulation. Annu Rev Microbiol 1994, 48:311-343.

3. Liu C, Gorby YA, Zachara JM, Fredrickson JK, Brown CF: Reduction kinetics of Fe (III), Co (III), U (VI), Cr (VI), and Tc (VII) in cultures of dissimilatory metal-reducing bacteria. Biotechnol Bioeng 2002, 80:637-649.

4. Hau HH, Gilbert A, Coursolle D, Gralnick JA: Mechanism and Consequences of anaerobic respiration of cobalt by Shewanella oneidensis strain MR-1. Appl Environ Microbiol 2008, 74:6880-6886.

5. Carpentier W, Sandra K, De Smet I, Brigé A, De Smet L, Van Beeumen J: Microbial reduction and precipitation of vanadium by Shewanella oneidensis. Appl Environ Microbiol 2003, 69:3636-3639.

6. Hau HH, Gralnick JA: Ecology and biotechnology of the genus Shewanella. Annu Rev Microbiol 2007, 61:237-258.

7. Kim BH, Kim HJ, Hyun MS, Park DH: Direct electrode reaction of Fe (III)reducing bacterium, Shewanella putrefaciens. J Microbiol Biotechnol 1999, 9:127-131.

8. Newton GJ, Mori S, Nakamura R, Hashimoto K, Watanabe K: Analyses of current-generating mechanisms of Shewanella loihica PV-4 and Shewanella oneidensis MR-1 in microbial fuel cells. Appl Environ Microbiol 2009, 75:7674-7681.

9. Ross DE, Flynn JM, Baron DB, Gralnick JA, Bond DR: Towards electrosynthesis in Shewanella: energetics of reversing the Mtr pathway for reductive metabolism. PLoS One 2011, 6:e16649.

10. Flynn JM, Ross DE, Hunt KA, Bond DR, Gralnick JA: Enabling unbalanced fermentations by using engineered electrode-interfaced bacteria. MBio 2010, 1:1-8.

11. Myers $\mathrm{CR}$, Nealson $\mathrm{KH}$ : Bacterial manganese reduction and growth with manganese oxide as the sole electron acceptor. Science 1988, 240:1319-1321.

12. Heidelberg JF, Paulsen IT, Nelson KE, Gaidos EJ, Nelson WC, Read TD, Eisen JA, Seshadri R, Ward N, Methe B, Clayton RA, Meyer T, Tsapin A, Scott J, Beanan M, Brinkac L, Daugherty S, DeBoy RT, Dodson RJ, Durkin a S, Haft DH, Kolonay JF, Madupu R, Peterson JD, Umayam LA, White O, Wolf AM, Vamathevan J, Weidman J, Impraim M, et al: Genome sequence of the dissimilatory metal ion-reducing bacterium Shewanella oneidensis. Nat Biotechnol 2002, 20:1118-1123.

13. Daraselia N, Dernovoy D, Tian Y, Borodovsky M, Tatusov R, Tatusova T: Reannotation of Shewanella oneidensis genome. OMICS 2003, 7:171-175.

14. Bretschger O, Obraztsova A, Sturm CA, Chang IS, Gorby YA, Reed SB, Culley DE, Reardon CL, Barua S, Romine MF, Zhou J, Beliaev AS, Bouhenni R, Saffarini D, Mansfeld F, Kim BH, Fredrickson JK, Nealson KH: Current production and metal oxide reduction by Shewanella oneidensis MR-1 wild type and mutants. Appl Environ Microbiol 2007, 73:7003-7012.

15. Shi L, Squier TC, Zachara JM, Fredrickson JK: Respiration of metal (hydr) oxides by Shewanella and Geobacter: a key role for multihaem c-type cytochromes. Mol Microbiol 2007, 65:12-20.

16. Gorby YA, Yanina S, McLean JS, Rosso KM, Moyles D, Dohnalkova A, Beveridge TJ, Chang IS, Kim BH, Kim KS, Culley DE, Reed SB, Romine MF, Saffarini DA, Hill EA, Shi L, Elias DA, Kennedy DW, Pinchuk G, Watanabe K, Ishii S, Logan B, Nealson KH, Fredrickson JK: Electrically conductive bacterial nanowires produced by Shewanella oneidensis strain MR-1 and other microorganisms. Proc Natl Acad Sci U S A 2006, 103:11358-11363. 
17. El-Naggar MY, Wanger G, Leung KM, Yuzvinsky TD, Southam G, Yang J, Lau WM, Nealson $\mathrm{KH}$, Gorby YA: Electrical transport along bacterial nanowires from Shewanella oneidensis MR-1. Proc Natl Acad Sci U S A 2010, 107:18127-18131.

18. Marsili E, Baron DB, Shikhare ID, Coursolle D, Gralnick JA, Bond DR Shewanella secretes flavins that mediate extracellular electron transfer. Proc Natl Acad Sci U S A 2008, 105:3968-3973.

19. von Canstein H, Ogawa J, Shimizu S, Lloyd JR: Secretion of flavins by Shewanella species and their role in extracellular electron transfer. Appl Environ Microbiol 2008, 74:615-623.

20. Watanabe K, Manefield M, Lee M, Kouzuma A: Electron shuttles in biotechnology. Curr Opin Biotechnol 2009, 20:633-641.

21. Okamoto A, Hashimoto K, Nealson KH, Nakamura R: Rate enhancement of bacterial extracellular electron transport involves bound flavin semiquinones. Proc Natl Acad Sci U S A 2013, 110:7856-7861.

22. Saffarini DA, Schultz R, Beliaev A: Involvement of cyclic AMP (cAMP) and CAMP receptor protein in anaerobic respiration of Shewanella oneidensis. J Bacterio/ 2003, 185:3668-3671.

23. Charania MA, Brockman KL, Zhang Y, Banerjee A, Pinchuk GE, Fredrickson JK, Beliaev AS, Saffarini DA: Involvement of a membrane-bound class III adenylate cyclase in regulation of anaerobic respiration in Shewanella oneidensis MR-1. J Bacterio/ 2009, 191:4298-4306.

24. Covington ED, Gelbmann CB, Kotloski NJ, Gralnick JA: An essential role for UshA in processing of extracellular flavin electron shuttles by Shewanella oneidensis. Mol Microbiol 2010, 78:519-532.

25. Kouzuma A, Meng XY, Kimura N, Hashimoto K, Watanabe K: Disruption of the putative cell surface polysaccharide biosynthesis gene SO3177 in Shewanella oneidensis MR-1 enhances adhesion to electrodes and current generation in microbial fuel cells. App/ Environ Microbiol 2010, 76:4151-4157

26. Tajima N, Kouzuma A, Hashimoto K, Watanabe K: Selection of Shewanella oneidensis MR-1 gene-knockout mutants that adapt to an electrode-respiring condition. Biosci Biotechnol Biochem 2011, 75:2229-2233.

27. Pernestig AK, Melefors O, Georgellis D: Identification of UvrY as the cognate response regulator for the BarA sensor kinase in Escherichia coli. $J$ Biol Chem 2001, 276:225-231.

28. Wei B, Shin S, LaPorte D, Wolfe AJ, Romeo T: Global regulatory mutations in csrA and rpoS cause severe central carbon stress in Escherichia coli in the presence of acetate. J Bacteriol 2000, 182:1632-1640.

29. Pernestig AK, Georgellis D, Romeo T, Suzuki K, Tomenius H, Normark S, Melefors O: The Escherichia coli BarA-UvrY two-component system is needed for efficient switching between glycolytic and gluconeogenic carbon sources. J Bacterio/ 2003, 185:843-853.

30. Binnenkade L, Lassak J, Thormann KM: Analysis of the BarA/UvrY TwoComponent System in Shewanella oneidensis MR-1. PLoS One 2011, 6: e23440.

31. Müller J, Shukla S, Jost KA, Spormann AM: The $m x d$ operon in Shewanella oneidensis MR-1 is induced in response to starvation and regulated by ArcS/ArcA and BarA/UvrY. BMC Microbiol 2013, 13:119.

32. Boyd A, Chakrabarty AM: Pseudomonas aeruginosa biofilms: role of the alginate exopolysaccharide. J Ind Microbiol 1995, 15:162-168.

33. Davey ME, Duncan MJ: Enhanced biofilm formation and loss of capsule synthesis: deletion of a putative glycosyltransferase in Porphyromonas gingivalis. J Bacteriol 2006, 188:5510-5523.

34. Korenevsky A, Beveridge TJ: The surface physicochemistry and adhesiveness of Shewanella are affected by their surface polysaccharides. Microbiology 2007, 153:1872-1883.

35. Tatusov RL, Fedorova ND, Jackson JD, Jacobs AR, Kiryutin B, Koonin EV Krylov DM, Mazumder R, Mekhedov SL, Nikolskaya AN, Rao BS, Smirnov S, Sverdlov AV, Vasudevan S, Wolf YI, Yin JJ, Natale DA: The COG database: an updated version includes eukaryotes. BMC Bioinformatics 2003, 4:41.

36. Fritsch PS, Urbanowski ML, Stauffer GV: Role of the RNA polymerase a subunits in MetR-dependent activation of metE and metH: important residues in the C-terminal domain and orientation requirements within RNA polymerase. J Bacteriol 2000, 182:5539-5550.

37. Weissbach $\mathrm{H}$, Brot $\mathrm{N}$ : Regulation of methionine synthesis in Escherichia coli. Mol Microbiol 1991, 5:1593-1597.

38. Niu C, Robbins CM, Pittman KJ, Osborn JL, Stubblefield BA, Simmons RB, Gilbert ES: LuxS influences Escherichia coli biofilm formation through autoinducer-2-dependent and autoinducer-2-independent modalities. FEMS Microbiol Ecol 2013, 83:778-791.
39. Learman DR, Yi H, Brown SD, Martin SL, Geesey GG, Stevens AM, Hochella MF: Involvement of Shewanella oneidensis MR-1 LuxS in biofilm development and sulfur metabolism. Appl Environ Microbio/ 2009, 75:1301-1307.

40. Gödeke J, Paul K, Lassak J, Thormann KM: Phage-induced lysis enhances biofilm formation in Shewanella oneidensis MR-1. ISME J 2011, 5:613-626.

41. Winzer K, Hardie KR, Burgess N, Doherty N, Kirke D, Holden MTG, Linforth R, Cornell KA, Taylor AJ, Hill PJ, Williams P: LuxS: its role in central metabolism and the in vitro synthesis of 4-hydroxy-5-methyl-3(2H)-furanone. Microbiology 2002, 148:909-922.

42. Pei D, Zhu J: Mechanism of action of S-ribosylhomocysteinase (LuxS). Curr Opin Chem Biol 2004, 8:492-497.

43. Vendeville A, Winzer K, Heurlier K, Tang CM, Hardie KR: Making "sense" of metabolism: autoinducer-2, LuxS and pathogenic bacteria. Nat Rev Microbiol 2005, 3:383-396.

44. Xavier KB, Bassler BL: LuxS quorum sensing: more than just a numbers game. Curr Opin Microbiol 2003, 6:191-197.

45. Chiang PK, Gordon RK, Tal J, Zeng GC, Doctor BP, Pardhasaradhi K, McCann PP: S-Adenosylmethionine and methylation. FASEB J 1996, 10:471-480.

46. Yeung ATY, Torfs ECW, Jamshidi F, Bains M, Wiegand I, Hancock REW, Overhage J: Swarming of Pseudomonas aeruginosa is controlled by a broad spectrum of transcriptional regulators, including MetR. J Bacterio/ 2009, 191:5592-5602.

47. Urbanowski ML, Stauffer GV: Role of homocysteine in metR-mediated activation of the metE and metH genes in Salmonella typhimurium and Escherichia coli. J Bacteriol 1989, 171:3277-3281.

48. Chatterjee J, Miyamoto CM, Zouzoulas A, Lang BF, Skouris N, Meighen EA: MetR and CRP bind to the Vibrio harveyi lux promoters and regulate luminescence. Mol Microbiol 2002, 46:101-111.

49. Plamann MD, Stauffer GV: Regulation of the Escherichia coli glyA gene by the metR gene product and homocysteine. J Bacterio/ 1989, 171:4958-4962.

50. Alexeyev MF, Shokolenko IN: Mini-Tn10 transposon derivatives for insertion mutagenesis and gene delivery into the chromosome of gram-negative bacteria. Gene 1995, 160:59-62.

51. Cheng S, Liu H, Logan BE: Increased power generation in a continuous flow MFC with advective flow through the porous anode and reduced electrode spacing. Environ Sci Technol 2006, 40:2426-2432.

52. Saltikov CW, Newman DK: Genetic identification of a respiratory arsenate reductase. Proc Natl Acad Sci U S A 2003, 100:10983-10988.

53. Watanabe K: Recent developments in microbial fuel cell technologies for sustainable bioenergy. J Biosci Bioeng 2008, 106:528-536.

54. Rosenberg M, Gutnick D, Rosenberg E: Adherence of bacteria to hydrocarbons: a simple method for measuring cell-surface hydrophobicity. FEMS Microbiol Lett 1980, 9:29-33.

55. Kouzuma A, Hashimoto K, Watanabe K: Influences of aerobic respiration on current generation by Shewanella oneidensis MR-1 in single-chamber microbial fuel cells. Biosci Biotechnol Biochem 2012, 76:270-275.

56. Kouzuma A, Hashimoto K, Watanabe K: Roles of siderophore in manganese-oxide reduction by Shewanella oneidensis MR-1. FEMS Microbiol Lett 2012, 326:91-98.

\section{doi:10.1186/1471-2180-14-190}

Cite this article as: Kouzuma et al.: Electrochemical selection and characterization of a high current-generating Shewanella oneidensis mutant with altered cell-surface morphology and biofilm-related gene expression. BMC Microbiology 2014 14:190.

\section{Submit your next manuscript to BioMed Central and take full advantage of:}

- Convenient online submission

- Thorough peer review

- No space constraints or color figure charges

- Immediate publication on acceptance

- Inclusion in PubMed, CAS, Scopus and Google Scholar

- Research which is freely available for redistribution 